\title{
Constraints on Torsion from Bounds on Lorentz Violation
}

\author{
V. Alan Kostelecký, ${ }^{1}$ Neil Russell, ${ }^{2}$ and Jay D. Tasson ${ }^{1}$ \\ ${ }^{1}$ Physics Department, Indiana University, Bloomington, Indiana 47405, USA \\ ${ }^{2}$ Physics Department, Northern Michigan University, Marquette, Michigan 49855, USA
}

(Received 10 January 2008; published 20 March 2008)

\begin{abstract}
Exceptional sensitivity to spacetime torsion can be achieved by searching for its couplings to fermions. Recent experimental searches for Lorentz violation are exploited to extract new constraints involving 19 of the 24 independent torsion components down to levels of order $10^{-31} \mathrm{GeV}$.
\end{abstract}

PACS numbers: 04.50.Kd, 04.20.-q, 11.30.Cp

In Einstein's general relativity, gravity is the curvature of spacetime and energy-momentum density is its source. Among the numerous alternative theories of gravity, one popular class of models involves introducing an additional warping of spacetime called torsion [1-3]. In many models, the torsion has spin density as its source. Some scenarios allow torsion waves to propagate through spacetime, in analogy with the traveling curvature waves that form gravitational radiation in general relativity. In the special class of "teleparallel" models, the curvature of spacetime is itself determined in terms of the torsion.

Theories extending general relativity via torsion are widely regarded as experimentally challenging to test because the effects of torsion typically are minuscule. Nature contains many sources of energy-momentum density sufficient to curve spacetime, such as stars and planets. However, sources of spin density strong enough to produce torsion effects are difficult to identify or create. Typical limits on torsion in the literature involve dynamical properties, being obtained from searches for spin-spin interactions or for torsion-mass effects [4].

In this Letter, we discuss an alternative approach to searching for torsion, based on the little-appreciated fact that background torsion violates effective local Lorentz invariance. The key point is that nonzero torsion over a region of spacetime establishes a preferred orientation for a freely falling observer, which is the defining criterion for local Lorentz violation [5]. Certain tests of Lorentz symmetry can therefore be reinterpreted as torsion searches. Related ideas have been suggested by Lämmerzahl [6] and Shapiro [2]. Here, we use the exquisite sensitivities recently achieved in Lorentz-violation searches to extract tight new constraints on torsion components, including many previously unbounded in the literature.

The recent surge of interest in tests of relativity stems from the realization that tiny violations of Lorentz symmetry could emerge from attempts to unify the known forces [7] and from the development of a comprehensive description of Lorentz and $C P T$ violation in the context of realistic effective field theory, called the standard-model extension (SME) [8]. The SME categorizes Lorentz violations by the mass dimension of the corresponding operator in the Lagrange density, which offers a simple measure of their expected size [9]. The physical effects are controlled by coefficients for Lorentz violation, and many experiments have been performed to measure them [10]. This work shows these experiments can be reinterpreted as searches for nonzero torsion. We note in passing that similar analyses might be relevant to nontorsion effects in other alternative gravity theories.

For the present Letter, we suppose that the physical gravitational field is described by a theory predicting a nonzero torsion field in the vicinity of Earth, and we seek model-independent constraints on the torsion insofar as possible. The specifics of the torsion field will depend on details of the theory and on the nature of the sources. To minimize model dependence, we take advantage of the fact that the dominant effects of nonzero torsion include various modifications of particle behavior arising from couplings to the torsion as a background. It is reasonable to approximate the leading-order torsion background as constant in a suitable reference frame relative to the torsion source. Analysis of subleading terms with torsion derivatives might conceivably yield additional results of interest, but this issue lies outside our present scope.

The constant-torsion background establishes a preferred orientation in the specified frame, producing effective local Lorentz violation. The Lagrange density describing particle couplings to background torsion can therefore be matched to the SME Lagrange density describing particle behavior in the presence of Lorentz violation. The match permits us to extract constraints on many torsion components from existing limits on SME coefficients for Lorentz violation. This procedure is sufficiently powerful to provide limits on models with both torsion and Lorentz violation and to distinguish the two in many cases $[5,11]$. However, since at present no compelling evidence exists for either Lorentz violation or torsion, our analysis here interprets experiments entirely in terms of torsion. In what follows, we summarize the calculations and results of this proposal. The conventions are those of Ref. [5].

The spacetime of general relativity is a Riemann manifold, determined by the Riemann curvature tensor $\tilde{R}_{\nu \alpha \beta}^{\mu}$. A spacetime with torsion is a Riemann-Cartan manifold, and it is specified by the generalized Riemann tensor $R^{\mu}{ }_{\nu \alpha \beta}$ and the torsion tensor $T^{\alpha}{ }_{\mu \nu}$. The tensor $R^{\mu}{ }_{\nu \alpha \beta}$ 
can be expressed as the sum of $\tilde{R}_{\nu \alpha \beta}^{\mu}$ and terms involving $T^{\alpha}{ }_{\mu \nu}$. Gravitational effects are negligible for our analysis of laboratory experiments, so we can assume $\tilde{R}_{\nu \alpha \beta}^{\mu} \approx 0$. The tensor $R_{\nu \alpha \beta}^{\mu}$ is then determined by the torsion and is nonzero only if the torsion is nonzero. Our analysis and results apply to most torsion theories predicting nonzero laboratory effects. Teleparallel models are exceptions, as they require $R_{\nu \alpha \beta}^{\mu}$ to vanish and $\tilde{R}_{\nu \alpha \beta}^{\mu}$ to be nonzero.

The torsion tensor obeys $T^{\alpha}{ }_{\mu \nu}=-T^{\alpha}{ }_{\nu \mu}$ and therefore has 24 independent components. It can be expanded as a sum involving three Lorentz-irreducible pieces,

$$
\begin{gathered}
T_{\alpha \mu \nu}=\frac{1}{3}\left(g_{\alpha \mu} T_{\nu}-g_{\alpha \nu} T_{\mu}\right)-\epsilon_{\mu \nu \alpha \beta} A^{\beta}+M_{\alpha \mu \nu}, \\
T_{\mu} \equiv g^{\alpha \beta} T_{\alpha \beta \mu}, \quad A^{\mu} \equiv \frac{1}{6} \epsilon^{\alpha \beta \gamma \mu} T_{\alpha \beta \gamma}, \\
M_{\alpha \mu \nu} \equiv \frac{1}{3}\left(T_{\alpha \mu \nu}+T_{\mu \alpha \nu}+T_{\mu} g_{\alpha \nu}\right)-\frac{1}{3}(\mu \leftrightarrow \nu) .
\end{gathered}
$$

The mixed-symmetry irreducible piece $M_{\alpha \mu \nu}$ satisfies the eight identities $g^{\alpha \beta} M_{\alpha \beta \mu}=0$ and $\epsilon^{\alpha \beta \gamma \mu} M_{\alpha \beta \gamma}=0$, leaving 16 independent combinations.

Our analysis sets constraints on torsion components through torsion couplings to standard-model fields. In most of the literature, the torsion is assumed to be minimally coupled through its appearance in covariant derivatives. However, nonminimal couplings are also possible. In this Letter, we focus on signals of torsion arising from both minimal and nonminimal couplings to fermions. Couplings to bosons, including photons and gravity, are discussed briefly below but yield constraints of lesser interest here.

The general behavior of a fermion of mass $m$ in a Riemann-Cartan spacetime with $\tilde{R}_{\nu \alpha \beta}^{\mu} \approx 0$ can be described by a Hermitian Lagrange density with arbitrary torsion couplings. We are interested in the constant-torsion approximation, for which the arbitrary torsion couplings can be replaced with background solutions to the torsion field equations taken as constant at leading order. The corresponding effective Lagrange density with all independent constant-torsion couplings of mass dimensions four and five can be written as

$$
\begin{aligned}
\mathcal{L}^{T} \supset & \frac{1}{2} i \bar{\psi} \gamma^{\mu} \overleftrightarrow{\partial}_{\mu} \psi-m \bar{\psi} \psi+\xi_{1}^{(4)} T_{\mu} \bar{\psi} \gamma^{\mu} \psi+\xi_{2}^{(4)} T_{\mu} \bar{\psi} \gamma_{5} \gamma^{\mu} \psi+\xi_{3}^{(4)} A_{\mu} \bar{\psi} \gamma^{\mu} \psi+\xi_{4}^{(4)} A_{\mu} \bar{\psi} \gamma_{5} \gamma^{\mu} \psi+\frac{1}{2} i \xi_{1}^{(5)} T^{\mu} \bar{\psi} \overleftrightarrow{\partial}_{\mu} \psi \\
& +\frac{1}{2} \xi_{2}^{(5)} T^{\mu} \bar{\psi} \gamma_{5} \overleftrightarrow{\partial}_{\mu} \psi+\frac{1}{2} i \xi_{3}^{(5)} A^{\mu} \bar{\psi} \overleftrightarrow{\partial}_{\mu} \psi+\frac{1}{2} \xi_{4}^{(5)} A^{\mu} \bar{\psi} \gamma_{5} \overleftrightarrow{\partial}_{\mu} \psi+\frac{1}{2} i \xi_{5}^{(5)} M_{\mu \nu}^{\lambda} \bar{\psi}_{\mathrm{\partial}_{\lambda}} \sigma^{\mu \nu} \psi+\frac{1}{2} i \xi_{6}^{(5)} T_{\mu} \bar{\psi} \overleftrightarrow{\partial}_{\nu} \sigma^{\mu \nu} \psi \\
& +\frac{1}{2} i \xi_{7}^{(5)} A_{\mu} \bar{\psi}_{\mathrm{\partial}_{\nu}} \sigma^{\mu \nu} \psi+\frac{1}{2} i \xi_{8}^{(5)} \epsilon^{\lambda \kappa \mu \nu} T_{\lambda} \bar{\psi} \overleftrightarrow{\partial}_{\kappa} \sigma_{\mu \nu} \psi+\frac{1}{2} i \xi_{9}^{(5)} \epsilon^{\lambda \kappa \mu \nu} A_{\lambda} \bar{\psi} \overleftrightarrow{\partial}_{\kappa} \sigma_{\mu \nu} \psi
\end{aligned}
$$

Here, nontorsion couplings are disregarded, and covariant derivatives have been approximated systematically. The values of $\xi_{j}^{(d)}$ depend on the torsion theory considered. For example, the special case of minimal coupling is recovered for $\xi_{4}^{(4)}=3 / 4$ with other couplings zero.

Each torsion component in $\mathcal{L}^{T}$ is constant, so it no longer has the particle Lorentz transformation properties [5] of the original torsion field. The theory therefore contains effective Lorentz violation. For example, $A_{\mu}$ now behaves as four scalars under particle Lorentz transformations. Moreover, since the number of indices on the irreducible torsion components is odd, all the terms in Eq. (2) violate effective $C P T$ symmetry. Laboratory experiments can therefore in principle discern different torsion signals for particles and antiparticles.

In $\mathcal{L}^{T}$, each constant-torsion field and its associated coupling constant can be reinterpreted as a constant coefficient for a fermion field operator having mass dimension three or four. With this reinterpretation, $\mathcal{L}^{T}$ can be matched to the Minkowski-spacetime limit of the fermion sector of the minimal SME [5], which includes terms of dimension four or less. In this sector, the coefficients for Lorentzviolation controlling $C P T$-odd effects are conventionally denoted $a_{\mu}, b_{\mu}, e_{\mu}, f_{\mu}$, and $g_{\mu \nu \alpha}=-g_{\nu \mu \alpha}$. The latter can be decomposed into components $g_{\mu}^{(T)}, g_{\mu}^{(A)}$, and $g_{\mu \nu \alpha}^{(M)}$ in analogy with Eq. (1). However, in the present context only the combination $b_{\mu}-m g_{\mu}^{(A)}$ and the mixed irreducible component $g_{\mu \nu \alpha}^{(M)}$ are independent observables at leading order $[5,8,12]$. Matching these combinations to irreducible torsion components gives

$$
\begin{aligned}
b_{\mu}-m g_{\mu}^{(A)} & =-\left(\xi_{2}^{(4)}-2 m \xi_{8}^{(5)}\right) T_{\mu}-\left(\xi_{4}^{(4)}-2 m \xi_{9}^{(5)}\right) A_{\mu}, \\
g_{\mu \nu \alpha}^{(M)} & =-2 \xi_{5}^{(5)} M_{\alpha \mu \nu},
\end{aligned}
$$

which fixes the correspondence between observable effective torsion couplings and minimal SME coefficients involving field operators up to dimension four. This correspondence could be extended to operators of arbitrary dimension using coefficients in the nonminimal SME.

To identify explicit constraints on torsion components, the reference frame in which the leading-order torsion background is constant must be identified. This frame depends on the underlying theory and factors such as the source of torsion. We first suppose that the constant-torsion approximation holds everywhere within the solar system, as might occur in models with torsion originating outside the solar system, perhaps on galactic or cosmological scales. It is then appropriate and convenient to adopt the Sun-centered celestial-equatorial frame with Cartesian coordinates $(T, X, Y, Z)$ that is widely used in reporting results of searches for Lorentz violation [13]. In this frame, the $Z$ axis is parallel to the Earth's rotation axis, and the $X$ axis points towards the vernal equinox.

Laboratory measurements of $b_{\mu}-m g_{\mu}^{(A)}$ and $g_{\mu \nu \alpha}^{(M)}$ have been reported in the Sun-centered frame for various fermion species [10]. Since torsion is a geometric phenomenon, its couplings can reasonably be assumed to be flavor 
independent. A search through the available measurements reveals that the sharpest sensitivities to torsion effects emerge from Zeeman measurements with a dual maser [14] and from studies of a spin-polarized torsion pendulum [15].

The results from the dual-maser experiment include six independent measurements of combinations of coeffi- cients for Lorentz violation involving the neutron, obtained by searching for modulations of the maser signal associated with the rotation of the Earth and its revolution about the Sun. Applying the match (3), we extract six constraints on combinations of torsion coefficients:

$$
\begin{array}{r}
\left|\left(\xi_{2}^{(4)}-2 m_{n} \xi_{8}^{(5)}\right) T_{X}+\left(\xi_{4}^{(4)}-2 m_{n} \xi_{9}^{(5)}\right) A_{X}+2 m_{n} \xi_{5}^{(5)} M_{T Y Z}\right|<1.6 \times 10^{-31} \mathrm{GeV}, \\
\left|\left(\xi_{2}^{(4)}-2 m_{n} \xi_{8}^{(5)}\right) T_{Y}+\left(\xi_{4}^{(4)}-2 m_{n} \xi_{9}^{(5)}\right) A_{Y}+2 m_{n} \xi_{5}^{(5)} M_{T Z X}\right|<1.9 \times 10^{-31} \mathrm{GeV}, \\
\left|\cos \eta\left[\left(\xi_{2}^{(4)}-2 m_{n} \xi_{8}^{(5)}\right) T_{T}+\left(\xi_{4}^{(4)}-2 m_{n} \xi_{9}^{(5)}\right) A_{T}+2 m_{n} \xi_{5}^{(5)} M_{Y Z X}\right]+2 m_{n} \xi_{5}^{(5)} \sin \eta\left(2 M_{T T X}-M_{Y Y X}\right)\right|<2.0 \times 10^{-27} \mathrm{GeV}, \\
\left|2 m_{n} \xi_{5}^{(5)}\left(M_{T T Z}+M_{X X Z}\right)\right|<3.6 \times 10^{-27} \mathrm{GeV}, \\
\left|\left(\xi_{2}^{(4)}-2 m_{n} \xi_{8}^{(5)}\right) T_{T}+\left(\xi_{4}^{(4)}-2 m_{n} \xi_{9}^{(5)}\right) A_{T}+2 m_{n} \xi_{5}^{(5)} M_{X Y Z}\right|<3.8 \times 10^{-27} \mathrm{GeV}, \\
\left|2 m_{n} \xi_{5}^{(5)}\left[\cos \eta\left(2 M_{T T Z}-M_{X X Z}\right)-\sin \eta\left(M_{T T Y}+M_{Z Z Y}\right)\right]\right|<1.6 \times 10^{-27} \mathrm{GeV},
\end{array}
$$

where $m_{n}$ is the neutron mass and $\eta \simeq 23.4^{\circ}$ is the inclination of the orbital plane of the Earth relative to the $X-Y$ plane.

The experiment with a spin-polarized torsion-pendulum sought potential Lorentz-violating signals modulated by the rotation of the Earth and a laboratory rotation of the pendulum. The results include measurements of three independent combinations of coefficients for Lorentz violation involving the electron [15]. Using Eq. (3), we obtain

$$
\begin{aligned}
& \left|\left(\xi_{2}^{(4)}-2 m_{e} \xi_{8}^{(5)}\right) T_{X}+\left(\xi_{4}^{(4)}-2 m_{e} \xi_{9}^{(5)}\right) A_{X}+2 m_{e} \xi_{5}^{(5)} M_{T Y Z}\right|<4.8 \times 10^{-31} \mathrm{GeV} \\
& \left|\left(\xi_{2}^{(4)}-2 m_{e} \xi_{8}^{(5)}\right) T_{Y}+\left(\xi_{4}^{(4)}-2 m_{e} \xi_{9}^{(5)}\right) A_{Y}+2 m_{e} \xi_{5}^{(5)} M_{T Z X}\right|<5.0 \times 10^{-31} \mathrm{GeV} \\
& \left|\left(\xi_{2}^{(4)}-2 m_{e} \xi_{8}^{(5)}\right) T_{Z}+\left(\xi_{4}^{(4)}-2 m_{e} \xi_{9}^{(5)}\right) A_{Z}+2 m_{e} \xi_{5}^{(5)} M_{T X Y}\right|<7.8 \times 10^{-30} \mathrm{GeV}
\end{aligned}
$$

Here, $m_{e}$ is the electron mass.

To gain some feeling for the scope of the sensitivities to galactic or cosmological torsion achieved in these experiments, it is useful to tabulate the results assuming that only one torsion component is nonvanishing at a time. Table I summarizes the best sensitivities achieved under this assumption. The first pair of columns displays results to torsion couplings involving dimension-three operators, while the second pair of columns shows results for the dimension-four case. The table reveals that 19 of the 24 torsion components are accessible to these two laboratory searches at this order. The results for $T_{\mu}$ and $M_{\alpha \mu \nu}$ are firsts, while those for $A_{\mu}$ improve existing ones in magnitude and detail. The sensitivities attained are of potential interest for model building, as a torsion magnitude of $10^{-27} \mathrm{GeV}$ is roughly comparable to that of the metric Laplacian on the surface of the Earth. If torsion is strictly minimally coupled, then only the components $A_{\mu}$ are constrained, and the best sensitivities achieved are

$$
\begin{aligned}
& \left|A_{T}\right|<2.9 \times 10^{-27} \mathrm{GeV} \simeq 1.5 \times 10^{-11} \mathrm{~m}^{-1}, \\
& \left|A_{X}\right|<2.1 \times 10^{-31} \mathrm{GeV} \simeq 1.1 \times 10^{-15} \mathrm{~m}^{-1}, \\
& \left|A_{Y}\right|<2.5 \times 10^{-31} \mathrm{GeV} \simeq 1.3 \times 10^{-15} \mathrm{~m}^{-1}, \\
& \left|A_{Z}\right|<1.0 \times 10^{-29} \mathrm{GeV} \simeq 5.3 \times 10^{-13} \mathrm{~m}^{-1} .
\end{aligned}
$$

In some of the torsion literature, the primary tensor is a linear combination of torsion components known as the contortion tensor $K_{\mu \alpha \nu}=-K_{\nu \alpha \mu}$. The contortion also has 24 independent components and can be defined as

$$
\begin{aligned}
K_{\mu \alpha \nu} & \equiv \frac{1}{2}\left(T_{\mu \alpha \nu}-T_{\nu \alpha \mu}+T_{\alpha \mu \nu}\right) \\
& \equiv \frac{1}{3}\left(g_{\mu \alpha} K_{\nu}^{(T)}-g_{\nu \alpha} K_{\mu}^{(T)}\right)-\epsilon_{\mu \alpha \nu \beta} K^{(A) \beta}+K_{\mu \alpha \nu}^{(M)} .
\end{aligned}
$$

The second equation displays the decomposition of the contortion into Lorentz-irreducible pieces, which are related to the Lorentz-irreducible torsion components by

$$
K_{\nu}^{(T)}=T_{\nu}, \quad K_{\nu}^{(A)}=\frac{1}{2} A_{\nu}, \quad K_{\mu \alpha \nu}^{(M)}=M_{\alpha \mu \nu} .
$$

The constraints (4) and (5) and the entries in Table I therefore also yield immediate results for the contortion.

The effective Lagrange density (2) is an expansion in only one fermion species. Torsion couplings involving more than one fermion, such as $T_{\mu} \bar{\psi}_{1} \gamma^{\mu} \psi_{2}$, could also be considered. The corresponding SME observables involve particle flavor changes, so relevant experiments would include meson oscillations or neutrino oscillations. However, the sensitivities to torsion achieved in such experiments are weaker than those displayed in Table I.

The sensitivities listed in Table I are obtained from fermion-torsion couplings. Other possibilities can also be considered. In the photon sector, the natural electromagnetic field strength $F_{\mu \nu}$ is the exterior derivative of the four-vector potential, and it has no torsion coupling. If instead $F_{\mu \nu}$ is defined via the covariant derivative, the 
TABLE I. Sensitivities to torsion components associated with SME operators of dimension three (first pair of columns) and dimension four (second pair). Modulus signs are suppressed. The third column indicates sensitivity to Sun-sourced $(S)$ and Earthsourced $(E)$ torsion (see text).

\begin{tabular}{|c|c|c|c|c|}
\hline Quantity & Sensitivity & Quantity & Sensitivity & Source \\
\hline$\overline{\xi_{2}^{(4)} T_{T}}$ & $10^{-27} \mathrm{GeV}$ & $\xi_{8}^{(5)} T_{T}$ & $10^{-27}$ & \\
\hline$\xi_{2}^{(4)} T_{X}$ & $10^{-31} \mathrm{GeV}$ & $\xi_{8}^{(5)} T_{X}$ & $10^{-31}$ & $S$ \\
\hline$\xi_{2}^{(4)} T_{Y}$ & $10^{-31} \mathrm{GeV}$ & $\xi_{8}^{(5)} T_{Y}$ & $10^{-31}$ & $S$ \\
\hline$\xi_{2}^{(4)} T_{Z}$ & $10^{-29} \mathrm{GeV}$ & $\xi_{8}^{(5)} T_{Z}$ & $10^{-26}$ & $S, E$ \\
\hline$\xi_{4}^{(4)} A_{T}$ & $10^{-27} \mathrm{GeV}$ & $\xi_{9}^{(5)} A_{T}$ & $10^{-27}$ & \\
\hline$\xi_{4}^{(4)} A_{X}$ & $10^{-31} \mathrm{GeV}$ & $\xi_{9}^{(5)} A_{X}$ & $10^{-31}$ & $S$ \\
\hline$\xi_{4}^{(4)} A_{Y}$ & $10^{-31} \mathrm{GeV}$ & $\xi_{9}^{(5)} A_{Y}$ & $10^{-31}$ & $S$ \\
\hline \multirow[t]{12}{*}{$\xi_{4}^{(4)} A_{Z}$} & $10^{-29} \mathrm{GeV}$ & $\xi_{9}^{(5)} A_{Z}$ & $10^{-26}$ & $S, E$ \\
\hline & & $\xi_{5}^{(5)} M_{T T X}$ & $10^{-27}$ & \\
\hline & & $\xi_{5}^{(5)} M_{T T Y}$ & $10^{-27}$ & \\
\hline & & $\xi_{5}^{(5)} M_{T T Z}$ & $10^{-28}$ & \\
\hline & & $\xi_{5}^{(5)} M_{X X Z}$ & $10^{-27}$ & \\
\hline & & $\xi_{5}^{(5)} M_{Y Y X}$ & $10^{-27}$ & \\
\hline & & $\xi_{5}^{(5)} M_{Z Z Y}$ & $10^{-27}$ & \\
\hline & & $\xi_{5}^{(5)} M_{T X Y}$ & $10^{-26}$ & $S, E$ \\
\hline & & $\xi_{5}^{(5)} M_{T Y Z}$ & $10^{-31}$ & $S$ \\
\hline & & $\xi_{5}^{(5)} M_{T Z X}$ & $10^{-31}$ & $S$ \\
\hline & & $\xi_{5}^{(5)} M_{X Y Z}$ & $10^{-27}$ & \\
\hline & & $\xi_{5}^{(5)} M_{Y Z X}$ & $10^{-27}$ & \\
\hline
\end{tabular}

resulting minimal torsion coupling violates $\mathrm{U}(1)$ gauge invariance. However, nonminimal torsion couplings to photons that preserve $U(1)$ gauge invariance can be introduced in an effective-field expansion in powers of $F_{\mu \nu}$. In the approximation of constant-torsion background, the corresponding leading-order terms lie in the nonminimal SME and are of dimension five, so the resulting constraints are expected to be of lesser interest. The best existing sensitivity to terms of this type comes from studies of birefringence in the cosmic microwave background [16], but this involves cosmological scales rather than laboratory ones. Couplings to other gauge bosons in the electroweak and strong sectors also lead to sensitivities of lesser interest. In the gravity sector, measurements have recently been obtained for some coefficients for Lorentz violation [17] that may imply bounds on curvature-torsion couplings, but these are also expected to be of lesser interest here.

The above results assume background torsion on the scale of the solar system with dominantly constant components in the Sun-centered Cartesian frame. On phenomenological grounds one can consider instead "Sun-sourced" torsion, having an approximate azimuthal symmetry centered on the Sun and with symmetry axis normal to the ecliptic plane. In this scenario, the torsion components in the corresponding coordinates are approximately constant over the Earth's orbit. As a result, the revolution of the
Earth about the Sun produces no modulation at first approximation, so the relevant bounds on torsion components become restricted to those extracted from Lorentzviolation searches involving the rotation of the Earth or a laboratory rotation of the apparatus. The corresponding results include the first two dual-maser measurements in Eq. (4) and the three torsion-pendulum ones in Eq. (5). The Sun-sourced torsion sensitivities attained are those in Table I marked with an $S$ in the final column.

In a similar vein, one can consider "Earth-sourced" torsion having an approximate azimuthal symmetry centered on the Earth and with symmetry axis along the Earth's rotation axis. In principle, comparison of data between laboratories at different latitudes could reveal effects varying with distance from the rotation axis, but at fixed latitude the torsion components are approximately constant in the corresponding coordinate system. This means neither the rotation nor the revolution of the Earth modulate torsion effects in a given laboratory, so Lorentzviolation searches for sidereal and annual variations are irrelevant. In effect, in a given laboratory, only measurements arising from rotations of the apparatus or direct comparisons of the behavior of particles and antiparticles can reveal torsion couplings of this type, so only the third constraint in Eq. (5) applies. The resulting sensitivities attained are those marked with an $E$ in Table I.

This work was supported in part by DOE Grant No. DEFG02-91ER40661 and by NASA Grant No. NAG3-2194.

[1] F. W. Hehl et al., Rev. Mod. Phys. 48, 393 (1976).

[2] I. L. Shapiro, Phys. Rep. 357, 113 (2002).

[3] R. T. Hammond, Rep. Prog. Phys. 65, 599 (2002).

[4] S. M. Carroll and G. B. Field, Phys. Rev. D 50, 3867 (1994); A.S. Belyaev, I. L. Shapiro, and M. A. B. do Vale, Phys. Rev. D 75, 034014 (2007).

[5] V. A. Kostelecký, Phys. Rev. D 69, 105009 (2004).

[6] C. Lämmerzahl, Phys. Lett. A 228, 223 (1997).

[7] V. A. Kostelecký and S. Samuel, Phys. Rev. D 39, 683 (1989); V. A. Kostelecký and R. Potting, Nucl. Phys. B359, 545 (1991).

[8] D. Colladay and V. A. Kostelecký, Phys. Rev. D 55, 6760 (1997); 58, 116002 (1998).

[9] V. A. Kostelecký and R. Potting, Phys. Rev. D 51, 3923 (1995).

[10] V. A. Kostelecký and N. Russell, arXiv:0801.0287.

[11] R. Bluhm and V. A. Kostelecký, Phys. Rev. D 71, 065008 (2005).

[12] B. Altschul, J. Phys. A 39, 13757 (2006).

[13] V. A. Kostelecký and M. Mewes, Phys. Rev. D 66, 056005 (2002); R. Bluhm et al., Phys. Rev. D 68, 125008 (2003).

[14] F. Canè et al., Phys. Rev. Lett. 93, 230801 (2004).

[15] B. R. Heckel et al., Phys. Rev. Lett. 97, 021603 (2006).

[16] V. A. Kostelecký and M. Mewes, Phys. Rev. Lett. 99, 011601 (2007).

[17] J. B. R. Battat, J. F. Chandler, and C. W. Stubbs, Phys. Rev. Lett. 99, 241103 (2007); H. Müller et al., Phys. Rev. Lett. 100, 031101 (2008). 